\title{
O impacto das fraturas dentárias classe IV na vida de crianças e adolescentes
}

\author{
The impact of class IV fractures in the life of children and adolescents
}

\author{
Maiara Ester Schappo* \\ Eduardo Mohr \\ Luiza Helena Almeida***
}

\section{Resumo}

Objetivo: foi avaliar o impacto da qualidade de vida de crianças e adolescentes com fraturas dentárias classe IV. Sujeitos e método: crianças e adolescentes com idades entre 7 e 14 anos foram entrevistados por meio de um questionário sobre dados sociodemográficos e aspectos do traumatismo alveolodentário. Para avaliação do impacto da condição bucal na qualidade de vida e na autopercepção de saúde, foi aplicada a versão brasileira do questionário Child Perceptions Questionnaire (CPQ): 6-10 nas crianças de 7 a 10 anos de idade e 11-14 nas crianças de 11 e 14 anos. Resultados: participaram 19 crianças com idades entre 11 a 14 anos. Os traumas foram restritos à fratura de tecidos duros (esmalte e dentina) sem exposição pulpar. Quando questionados sobre como bateram o dente, justificaram das seguintes formas: $63,1 \%$ (12) em colisão com pessoa ou objeto; $15,7 \%$ (3) em queda da própria altura; 5,2\% (1) em práticas esportivas; $5,2 \%$ (1) em situações de violência; $5,2 \%$ (1) em acidente de carro, moto ou bicicleta; e $5,2 \%$ (1) em outras circunstâncias. A maioria dos traumas ocorreu na rua $(68,4 \%, 13)$ e em casa $(10,5 \%$, 2). Sobre $o$ aspecto estético antes do tratamento, $42,1 \%$ dos entrevistados consideravam seus dentes mais ou menos, 31,5\%, ruins, $15,7 \%$, bons, e $10,5 \%$, muito bons. Após o tratamento restaurador, esse mesmo aspecto foi realizado, e as respostas foram: $21 \%$ consideravam seus dentes mais ou menos, 5,2\%, ruins, $26,3 \%$, bons, e 47,3\%, muito bons. Conclusão: crianças e adolescentes que recebem tratamento imediato obtêm resultados favoráveis, minimizando traumas psicológicos e melhorando sua qualidade de vida.

Palavras-chave: Adolescentes. Crianças. Qualidade de vida. Dente decíduo. Traumatismos dentários.

\section{Introdução}

O traumatismo alvéolo dentário (TAD) pode ocorrer desde uma pequena fratura no esmalte até a perda definitiva do elemento dentário. Em sua maioria, os traumas são em regiões anteriores, interferindo não apenas na função, mas também na estética, passando a ter valor especial na vida de crianças e adolescentes. Os incisivos centrais, além de estarem na região anterior, apresentam particularidades, como overjet incisal (protrusão dos dentes ântero-superiores nos pacientes classe II de Angle divisão I) e selamento labial reduzido ${ }^{1}$.

Concomitante a isso, a prática de esportes sem cuidados prévios, o aumento dos níveis de violência, acidentes de trânsito, e a maior participação das crianças em atividades esportivas contribuem para transformar o TAD em problema de saúde pública emergente ${ }^{2}$. Além do comprometimento estético, gera ansiedade e incapacidades físicas, como fonação e mastigação, interferindo diretamente na qualidade de vida da população infantil e juvenil ${ }^{3}$, criando constrangimentos que fazem com que crianças e adolescentes evitem sorrir. $O$ fato de o TAD acometer com maior prevalência os dentes anteriores faz desse problema uma das maiores causas de urgências odontológicas ${ }^{4}$.

Pouca atenção, todavia, tem sido endereçada ao tratamento das lesões traumáticas, especialmente às fraturas que levam ao comprometimento estético. No Brasil, um estudo em Joaçaba, SC, mostrou a necessidade de tratamento em $88,0 \%$ dos casos. Isso representa uma frequência relativa de 9,8

\footnotetext{
Acadêmica de Odontologia, Centro de Saúde, Faculdade Avantis, Balneário Camboriú, Santa Catarina, Brasil.

Acadêmica de Odontologia, Centro de Saúde, Faculdade Avantis, Balneário Camboriú, Santa Catarina, Brasil.

** Professora coordenadora da área de Odontopediatria, Centro de Saúde, Faculdade Avantis, Santa Catarina, Brasil.
} 
dentes com necessidade de restaurações adesivas a cada mil incisivos analisados ${ }^{5}$. Nas situações em que já existe o dano, é preciso não negligenciar o tratamento restaurador e/ou reabilitador, buscando solucionar ou minimizar as consequências de um trauma, para que não haja danos negativos na qualidade de vida de crianças e adolescentes ${ }^{3}$.

Dessa forma, o objetivo deste estudo foi avaliar o impacto negativo das fraturas dentárias classe IV na qualidade de vida de crianças e adolescentes, por meio da aplicação de um questionário nos atendimentos prestados na Clínica Integrada de Odontopediatria do curso de Odontologia da Faculdade Avantis, Balneário Camboriú, Santa Catarina, Brasil.

\section{Sujeitos e método}

Trata-se de um estudo observacional, prospectivo, do tipo antes e depois ${ }^{6}$, previamente aprovado pelo comitê de ética em pesquisa da Faculdade Avantis, sob o Parecer n⿳0 2.077.465, atendendo aos requisitos da Resolução do Conselho Nacional de Saúde nº 466/96.

Os pesquisadores dirigiram-se às mães de crianças e adolescentes na faixa etária entre 7 e 14 anos que tivessem indicação de tratamento restaurador para fratura classe IV, em decorrência de traumatismo dentário, configurando-se uma amostra por conveniência. Após explicar os objetivos da pesquisa e obter o consentimento na participação dos filhos, foram coletados do prontuário dados referentes a sexo, idade, escolaridade materna, renda e estrutura familiares.

Foi realizada a entrevista com as crianças e os adolescentes por meio de questionário. Dados sobre o local de ocorrência e a etiologia do traumatismo alveolodentário, além de informações sobre higiene bucal, foram coletados. Para avaliação do impacto da condição bucal na qualidade de vida e na autopercepção de saúde, foi aplicada a versão brasileira do questionário Child Perceptions Questionnaire (CPQ): 6-10 às crianças de 7 a 10 anos e 11-14 às crianças de 11 e 14 anos. O CPQ é um instrumento de avaliação da qualidade de vida relacionada à saúde bucal, desenvolvido por Locker et al. ${ }^{7}$ (2002). O questionário sobre a qualidade de vida foi aplicado em dois momentos: antes e depois do tratamento restaurador. Os dados foram digitados em planilha do programa Microsoft Excel, e foi realizada estatística descritiva.

\section{Resultados}

A amostra foi constituída de 19 crianças com faixa etária entre 11 e 14 anos. O Quadro 1 mostra a distribuição da amostra de acordo com os dados sociodemográficos.
Quadro 1 - Distribuição de crianças e adolescentes, de acordo com as características sociodemográficas

\begin{tabular}{|c|c|c|c|}
\hline \multicolumn{2}{|r|}{ Variáveis } & $N$ & $\%$ \\
\hline Gênero & $\begin{array}{l}\text { Masculino } \\
\text { Feminino }\end{array}$ & $\begin{array}{c}13 \\
6\end{array}$ & $\begin{array}{l}68,4 \% \\
31,6 \%\end{array}$ \\
\hline $\begin{array}{l}\text { Núcleo } \\
\text { familiar }\end{array}$ & $\begin{array}{l}\text { Pais } \\
\text { Pai } \\
\text { Mãe e padrasto }\end{array}$ & $\begin{array}{l}16 \\
2 \\
1\end{array}$ & $\begin{array}{r}84,2 \% \\
10,5 \% \\
5,3 \%\end{array}$ \\
\hline $\begin{array}{l}\text { Escolaridade } \\
\text { materna }\end{array}$ & $\begin{array}{l}\text { Não estudou } \\
\text { Ensino Fundamental incompleto } \\
\text { Ensino Médio incompleto } \\
\text { Ensino superior incompleto }\end{array}$ & $\begin{array}{l}1 \\
12 \\
3 \\
3\end{array}$ & $\begin{array}{r}5,3 \% \\
63,1 \% \\
15,8 \% \\
15,8 \%\end{array}$ \\
\hline $\begin{array}{l}\text { Renda } \\
\text { familiar }\end{array}$ & $\begin{array}{l}1 \text { salário mínimo } \\
2 \text { salários mínimos } \\
\text { Acima de } 3 \text { salários mínimos }\end{array}$ & $\begin{array}{l}2 \\
2\end{array}$ & $\begin{array}{l}79,0 \% \\
10,5 \% \\
10,5 \%\end{array}$ \\
\hline
\end{tabular}

Fonte: autores.

Quanto aos hábitos de higiene, quando questionados sobre a escovação de dentes, 84,2\%(16) disseram escovar os dentes 3 vezes ao dia, e $15,7 \%$ (3) 2 vezes ao dia, sendo que todos utilizavam creme dental. Para o relato de sangramento durante a escovação, 68,4\% (13) não apresentavam, 21\% (4) apresentavam sempre, e 10,5\% apresentavam (2) às vezes.

Todos os participantes bateram o dente, e todos os traumas foram restritos a fratura de tecidos duros (esmalte e dentina) sem exposição pulpar. A maioria dos traumas ocorreu na rua $68,4 \%$ (13) e no lar 10,5\% (2), também houve casos na escola, entre outros lugares. O Gráfico 1 apresenta a distribuição da amostra segundo a forma como bateram os dentes.

Gráfico 1 - Distribuição da amostra conforme a forma como bateram o dente

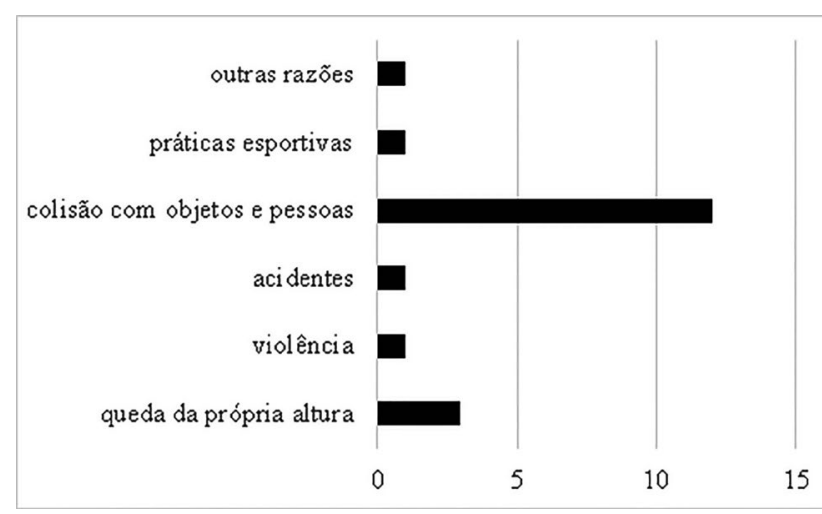

Fonte: autores.

Como fator de risco para o trauma, a maioria, 73,6\% (14), apresentou sobressaliência. Quando questionados sobre atendimento odontológico após o trauma, afirmaram não ter procurado tratamento. A respeito de relato de dor, 10 crianças não sen- 
tiram dor nos últimos seis meses, as demais, sim. Para a mesma pergunta nos últimos quatro meses, 8 não sentiram dor, 11, sim. Apenas 2 crianças $(10,5 \%)$ relataram medo de ir ao dentista para tratamento.

O Gráfico 2 aponta a autopercepção acerca dos aspectos estéticos antes e depois o tratamento. $\mathrm{O}$ Quadro 2 mostra a distribuição da amostra de acordo com as respostas dadas ao questionário sobre qualidade de vida, aplicado antes e depois o tratamento restaurador.
Gráfico 2 - Percepção dos dentes antes e depois o tratamento restaurador

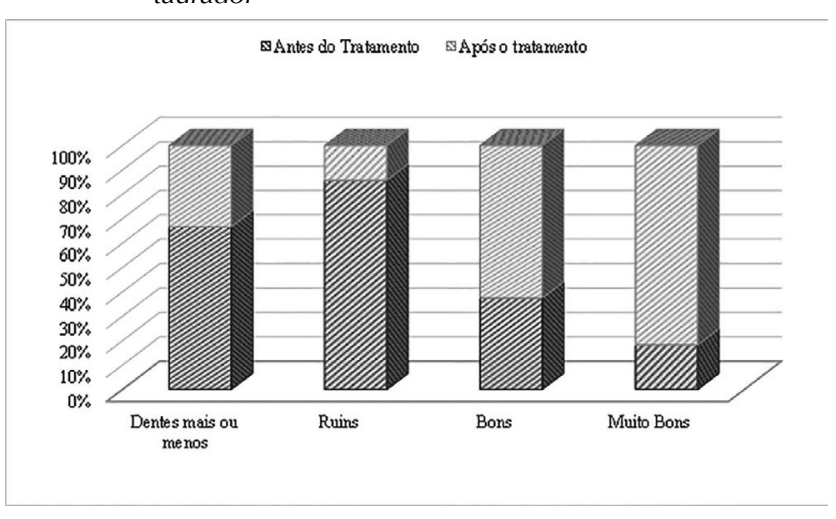

Fonte: autores.

Quadro 2 - Distribuição da amostra segundo respostas dadas ao questionário de qualidade de vida, aplicado antes e depois do tratamento restaurador

\begin{tabular}{|c|c|c|c|c|c|c|c|c|c|c|}
\hline & \multicolumn{5}{|c|}{ Antes } & \multicolumn{5}{|c|}{ Depois } \\
\hline & Nunca & $\begin{array}{l}1 \text { ou } 2 \\
\text { vezes }\end{array}$ & $\begin{array}{l}\text { Algumas } \\
\text { vezes }\end{array}$ & $\begin{array}{l}\text { Frequente- } \\
\text { mente }\end{array}$ & $\begin{array}{l}\text { Todos os } \\
\text { dias }\end{array}$ & Nunca & $\begin{array}{l}1 \text { ou } 2 \\
\text { vezes }\end{array}$ & $\begin{array}{l}\text { Algumas } \\
\text { vezes }\end{array}$ & $\begin{array}{l}\text { Frequente- } \\
\text { mente }\end{array}$ & $\begin{array}{l}\text { Todos os } \\
\text { dias }\end{array}$ \\
\hline $\begin{array}{l}\text { Chateado por causa } \\
\text { dos dentes }\end{array}$ & 7 & 2 & 6 & 4 & 0 & 11 & 2 & 6 & 0 & 0 \\
\hline $\begin{array}{l}\text { Sentiu-se } \\
\text { envergonhado }\end{array}$ & 10 & 0 & 3 & 0 & 6 & 15 & 0 & 0 & 3 & 1 \\
\hline $\begin{array}{l}\text { Sentiu-se } \\
\text { preocupado }\end{array}$ & 14 & 0 & 2 & 3 & 0 & 17 & 0 & 2 & 0 & 0 \\
\hline $\begin{array}{l}\text { Evitou sorrir ou dar } \\
\text { risada }\end{array}$ & 7 & 5 & 4 & 0 & 3 & 16 & 2 & 0 & 0 & 1 \\
\hline Não era tão bonito & 9 & 0 & 0 & 6 & 4 & 14 & 2 & 3 & 0 & 0 \\
\hline $\begin{array}{l}\text { Quantas vezes } \\
\text { fizeram gozação ou } \\
\text { colocaram apelidos }\end{array}$ & 3 & 0 & 7 & 8 & 1 & 9 & 3 & 2 & 4 & 1 \\
\hline
\end{tabular}

Fonte: autores.

\section{Discussão}

O TAD é considerado um problema de saúde pública, e seu número de ocorrências vem aumentando significativamente, sendo acometidos dois a cada três crianças ou adolescentes ${ }^{3}$. Os estudos apontam que a maioria das crianças que sofrem traumatismo é do sexo masculino, o que corrobora com os achados deste estudo. $\mathrm{O}$ sexo masculino apresenta maior incidência pelo fato de tais indivíduos estarem mais vinculados à prática de atividades esportivas mais radicais. No entanto, os resultados apontam que 0 fator causal mais encontrado é a colisão com pessoa ou objeto. Mas tal abordagem precisa ser repensada, pois, com o empoderamento feminino, nota-se um aumento de mulheres escolhendo práticas esportivas ${ }^{8}$.

Além das práticas esportivas, overjet incisal e falta de selamento labial são os principais fatores predisponentes, que, associados a fatores causais, como colisão com objetos ou pessoas, quedas da própria altura e acidentes, podem acometer trauma dentário ${ }^{9}$. Para este estudo, a maioria $(74,6 \%)$ apresentava sobressaliência como fator predisponente.
O traumatismo encontrado restringiu a fratura aos tecidos duros, esmalte e dentina. Por ser em dentes anteriores, o apelo estético poderia ser bastante importante e levaria à busca por tratamento odontológico. No entanto, observou-se que todos os participantes não procuraram o dentista para tratamento. Uma das razões para essa situação poderia ser o medo frente ao atendimento odontológico, no entanto, apenas duas crianças $(10,5 \%)$ relataram ter medo de ir ao dentista.

A dificuldade da busca por atendimento no serviço público de saúde pode ter contribuído para a situação, uma vez que razões financeiras impedem o tratamento no atendimento particular, já que a maioria dos pais tem apenas ensino fundamental incompleto e renda familiar variando de 1 a 2 salários mínimos. Além do mais, por serem traumas de complexidade menor, a família acaba não dando a atenção necessária, motivo pelo qual o reparo não é imediato ${ }^{5}$.

Estudos apontam que traumas leves não impactam negativamente a qualidade de vida de crianças ${ }^{10,11}$, outra razão para a falta de procura por atendimento. Além disso, por se tratar apenas de fratura de esmalte e dentina, sem exposição pulpar, 
a dor ou a sensibilidade é minimizada, e a busca pelo atendimento, protelada. Mais da metade dos participantes respondeu que, nos últimos 4 meses, sentiu dor, e tal fator poderia ser motivo de procura por atendimento. No entanto, não é possível saber se a dor foi em decorrência do trauma ou de outras lesões de acometimento pulpar ou periapical, bem como fazer uma comparação fidedigna com o momento do traumatismo. Bendo et al. ${ }^{12}$ (2014) relataram que, em casos de fraturas mais complexas, envolvendo dentina e comprometimento pulpar, aumentam em 2,4 vezes as chances do impacto negativo e a busca pelo tratamento.

Apesar de não terem buscado o atendimento para restaurar o dano perdido, sabemos que os TADs podem impactar negativamente na qualidade de vida de crianças e adolescentes ${ }^{13-15}$. Crianças que sofreram trauma e não receberam tratamento restaurador tendem a relatar maior impacto, como dificuldade em se alimentar e fazer a higiene bucal, e acabam demonstrando mais vergonha de sorrir, sentem-se chateadas, o que interfere na fonação, quando comparadas àquelas que não sofreram trau$\mathrm{ma}^{3,13,16,17}$.

Os incisivos superiores são os dentes mais acometidos, como encontrado neste estudo. Ao observar os resultados para as perguntas "já sentiu vergonha?", "evitou sorrir?", "não era tão bonito como os outros?", respostas positivas foram dadas por muitas crianças antes do tratamento; já, depois dele, ao serem indagadas novamente pelas mesmas questões, os resultados foram minimizados.

Em se tratando de pacientes adolescentes, uma vez que a amostra constituiu a faixa etária de 11 a 14 anos, este apelo estético é ainda mais valorizado, pois é uma das etapas da vida com maior influência nesse sentido. É um ciclo em que ocorrem as mais diversas mudanças biológicas e psicológicas; tais aspectos podem ser potencializados pelo TAD, principalmente no âmbito estético, em razão desse período mais vulnerável da vida ${ }^{18}$.

Assim, as mudanças que ocorrem, principalmente com relação à saúde bucal, afetam a qualidade de vida e o bem-estar, pois é um período em que estão começando a se descobrir e a moldar sua identidade ${ }^{19}$. O questionário Early Childhood Oral Health Impact Scale (Ecohis) para este grupo de pessoas permitiu verificar que, após o tratamento restaurador, houve mudanças nas respostas dadas por crianças e adolescentes, com relatos sobre "ter dentes ruins" evoluirem para "dentes bons" ou "muito bons". $\mathrm{Na}$ abordagem mais positiva, "dentes muito bons", houve um ganho de $36,8 \%$, com melhora de autoestima antes e depois do tratamento. É de suma importância explorar a qualidade de vida relacionada ao traumatismo dentário com a família e profissionais da área, uma vez que parece notável o impacto negativo deste aspecto na qualidade de vida, com intuito até mesmo de preparar profissionais para buscar estratégias de promoção de saúde, a fim de minimizar, ou solucionar, os casos de trauma $a^{1-3,8,13,18-20}$.
Negligenciar o tratamento pode aumentar em até quatro vezes o impacto na qualidade de vida, no sorriso, na alimentação e no convívio com outras pessoas $^{3,15,18,21}$. Para tratamentos de fraturas de esmalte e dentina, como nos casos deste estudo, em sua grande maioria, apenas restaurações adesivas são necessárias como solução para os traumas. No entanto, é notável o despreparo de alguns profissionais frente à situação de urgência, principalmente em casos de traumas mais complexos. Paiva et al. ${ }^{20}$ (2013) mostram que 59\% dos dentes que receberam tratamento não estavam em boas condições devido à falta de conhecimento e preparo de cirurgiões-dentistas frente às urgências relacionadas ao trauma. Atendimentos sem correto diagnóstico e execução de um tratamento rápido, muitas vezes, podem levar à perda permanente do dente com fratura, principalmente a longo prazo. Mesmo dentes com pequenas fraturas de esmalte necessitam tratamento ${ }^{1-3,8,13,18-20}$.

Nestes achados, no que diz respeito à estética, após o tratamento recebido, $84,2 \%$ das crianças acometidas passaram a sorrir. Justamente por ser de menor complexidade, é necessária a realização de campanhas ou de estudos direcionados aos cirurgiões-dentistas, para que estejam melhor preparados para atender e resolver casos de trauma e, assim, evitar ou minimizar possíveis danos ${ }^{1}$. Medidas preventivas e cuidados prévios à prática de esportes, como utilização de abridores de boca, ações educativas para ciclovias, estratégias para a redução de acidentes de trânsito, cuidados preventivos dos responsáveis por crianças pequenas, são alternativas plausíveis, para evitar os traumatismos dentários e, consequentemente, a redução deste problema de saúde pública.

Dentro das limitações deste estudo, pelo fato de a amostra ser por conveniência, os resultados não podem ser extrapolados para toda a população de crianças e adolescentes do município. Foram importantes ter um panorama sobre os aspectos da qualidade de vida para esta amostra e perceber que, mesmo havendo relatos na literatura de que traumas menores não impactam na vida das crianças, houve mudança considerável dos relatos de autopercepção estética antes e depois dos procedimentos simples de reabilitação.

\section{Conclusão}

O trauma dental tem forte impacto negativo e interfere diretamente na qualidade de vida de crianças e adolescentes. A busca por atendimento ainda é baixa, quando comparada ao preparo de cirurgiões-dentistas frente às urgências e ao manejo dos pacientes. Crianças e adolescentes que recebem tratamento imediato obtêm resultados favoráveis, minimizando traumas psicológicos e melhorando sua qualidade de vida. 


\section{Abstract}

Objective: evaluate the impact of the quality of life of children and adolescents with class IV dental fractures. Subjects and method: children and adolescents aged 7 to 14 years were interviewed through a questionnaire on sociodemographic data and aspects of alveolodentary trauma. The Brazilian version of the Child Perceptions Questionnaire (CPQ) questionnaire 6-10 was used to assess the impact of oral conditions on quality of life and self-perception of health in children aged 7-10 years and 11-14 in children aged 11 and 14 years. Results: nineteen children were included, and the age ranged from 11 to 14 years. Traumas were restricted to fracture of hard tissues (enamel and dentin) without pulp exposure. When questioned about how they hit the tooth, $63.1 \%$ (12) answered that in a collision with another person or object, $15.7 \%$ (3) that in falling of the same height, $5.2 \%$ (1) in sports, $5.2 \%$ (1) for violence, $5.2 \%$ (1) in a car, motorcycle or bicycle accident and 5.2\% (1) for other reasons. Most traumas occurred on the street $(68.4 \%, 13)$, and at home $(10.5 \%, 2)$. Regarding the aesthetic aspect before treatment, $42.1 \%$ of the interviewees considered their teeth more or less, $31.5 \%$ bad, $15.7 \%$ good and $10.5 \%$ very good. After the restorative treatment the same question was asked and the answers were: $21 \%$ considered their teeth more or less, $5.2 \%$ bad, $26.3 \%$ good and $47.3 \%$ very good. Conclusion: children and adolescents receiving immediate treatment obtain favorable results, minimizing psychological trauma and improving their quality of life.

Keywords: Adolescents. Children. Dental trauma. Primary teeth. Quality of life.

\section{Referências}

1. Mota LQ, Largino AGR, Lima MGC, Farias JFG, Silva ALA, Farias FFG. Estudo do Traumatismo Dentário em Escolares do Município de João Pessoa, PB, Brasil. Pesq Bras Odontoped $2011 ; 11(2): 217-22$.

2. Traebert J, Almeida IC, Garghetti C, Marcenes W. Prevalência, necessidade de tratamento e determinantes do traumatismo dentário na dentição permanente de escolares de 11 a 13 ano de idade. Cad Saúde Pública 2004; 20(2):403-10.

3. Antunes LA, Leão AT, Maia LC. The impact of dental trauma on quality of life of children and adolescents: a critical review and measurement instruments. Cienc Saúde Colet 2012; 17(12):3417-24.

4. Traebert J, Almeida ICS, Marcenes W. Aetiology of traumaticdental injuries in 11 to 13 -year-old schoolchildren. Oral Health Prev Dent 2003; 1(4):317-23.

5. Traebert J, Marcon KB, Lacerda JT. Prevalência de traumatismo dentário e fatores associados em escolares do município de Palhoça (SC). Cien Saude Colet 2010; 15(1):1849-55.

6. Antunes JLF, Peres MA. Fundamentos de Odontologia - Epidemiologia da saúde bucal. São Paulo: Guanabara Koogan; 2006.

7. Locker D, Jokovic A, Stephens M, Kenny D, Tompson B, Guyatt G. Family impact of child oral and oro-facial conditions. Community Dent Oral Epidemiol 2002; 30(6):438-48.

8. Traebert J, Claudino D. Epidemiologia do Traumatismo Dentário em Crianças: a Produção Científica Brasileira. Pesq Bras Odontoped 2012; 12(2):263-72.
9. Siluvai S, Kshetrimayum N, Reddy CV, Siddanna S, Manjunath M, Rudraswamy S. Malocclusion and related quality of life among 13- to 19-year-old students in Mysore City - a cross-sectional study. Oral Health Prev Dent 2015; 13(2):135-41.

10. Abanto J, Tsakos G, Paiva SM, Carvalho TS, Raggio DP, Bönecker M. Impact of dental caries and trauma on quality of life among 5- to 6-year-old children: perceptions of parents and children. Community Dent Oral Epidemiol 2014; 42(5):385-94

11. Viegas CM, Paiva SM, Carvalho AC, Scarpelli AC, Ferreira FM, Pordeus IA. Influence of traumatic dental injury on quality of life of Brazilian preschool children and their families. Dent Traumatol 2014; 30(5):338-47.

12. Bendo CB, Paiva SM, Varni JW, Vale MP. Oral health-related quality of life and traumatic dental injuries in Brazilian adolescents. Community Dent Oral Epidemiol 2014; 42(3):216-23.

13. Cortes MI, Marcenes W, Sheiham A. Impact of traumatic injuries to the permanent teeth on the oral health-related quality of life in 12-14-year-old children. Community Dent Oral Epidemiol 2002; 30(3):193-8.

14. Freire-Maia FB, Auad SM, Abreu MH, Sardenberg F, Martins MT, Paiva SM et al. Oral Health-Related Quality of Life and Traumatic Dental Injuries in Young Permanent Incisors in Brazilian Schoolchildren: A Multilevel Approach. PLoS One 2015; 10(8):1-18.

15. Ramos-Jorge J, Paiva SM, Tataounoff J, Pordeus IA, Marques LS, Ramos-Jorge ML. Impact of treated/untreated traumatic dental injuries on quality of life among Brazilian schoolchildren. Dent Traumatol 2014; 30(1):27-31.

16. Antunes LS, Debossan PF, Bohrer LS, Abreu FV, Quintanilha LE, Antunes LA. Impact of traumatic dental injury on the quality-of-life of children and adolescents: a case-control study. Acta Odontol Scand 2013; 71(5):1123-8.

17. Vieira-Andrade RG, Siqueira MB, Gomes GB, D'Avila S, Pordeus IA, Paiva SM et al. Impact of traumatic dental injury on the quality of life of young children: a case-control study. Int Dent J 2015; 65(5):261-8.

18. Oliveira DC, Pereira PN, Ferreira FM, Paiva SM, Fraiz FC. Impacto relatado das alterações bucais na qualidade de vida de adolescentes: revisão sistemática. Pesq Bras Odontoped 2013; 13(1):123-9.

19. Severo IF, Colares V, Rosenblatt A. Abordagem psicológica do adolescente pelos cirurgiões-dentistas da cidade do Recife. Rev Iberoam Odontopediatr Odontol Bebê 2004; 7(38):377-86

20. Paiva PCP, Paiva HN, Jorge KO, Filho PMO. Estudo transversal em escolares de 12 anos de idade sobre a necessidade de tratamento, etiologia e ocorrência de traumatismo dentário em Montes Claros, Brasil. Arq Odontol 2013; 49(1):19-25.

Endereço para correspondência:

Luiza Helena Almeida

Centro de Saúde - Odontologia

Faculdade Avantis

Av. Marginal Leste, 3600, Estados

CEP 88339-125, Balneário Camboriú, SC, Brasil

Telefone: (47) 99264-9992

E-mail: luizahelenadentista@hotmail.com

Recebido: 05/11/17. Aceito: 26/11/17. 